\title{
Special issue: societal aspects of synthetic biology
}

\author{
Markus Schmidt
}

Received: 14 August 2009/Accepted: 17 August 2009

(C) The Author(s) 2009. This article is published with open access at Springerlink.com

For this special issue I brought together a highly interesting group of people, all dealing with different aspects of synthetic biology and its societal impact. Contributions by 31 (co-)authors led to 15 papers dealing with: ethics, theology, biosafety, biosecurity, governance strategies, the role of social scientists, the bioeconomy, epistemological question on the program and the machine, public perception of synthetic biology and the role of the media.

Readers of SSBJ already know that synthetic biology (SB) is the design and construction of new biological systems not found in nature. It aims at creating novel organisms for practical purposes but also at gaining insights into living systems by re-constructing them. SB as a scientific and engineering field currently includes the following subfields (Schmidt et al. 2009):

- Engineering DNA-based biological circuits, including but not limited to standardized biological parts;

- Defining a minimal genome/minimal life (top-down approach);

- Constructing so-called protocells, i.e. living cells, from scratch (bottom-up approach);

- Creating orthogonal biological systems based on a biochemistry not found in nature; and

- Gene and genome synthesis.

With many anticipated benefits and a high impact on society, the societal implications are becoming increasingly prominent. It is therefore crucial that the societal dimensions develop side by side with the field. I am

M. Schmidt $(\bowtie)$

Organisation for International Dialogue and Conflict

Management, Vienna, Austria

e-mail: markus.schmidt@idialog.eu convinced that this special issue will help to explore the societal issues of SB.

Those who follow the press coverage on SB have for sure come across astonishing headlines such as "First artificial life within months" or "Artificial life could be created within FIVE years, experts claim". Hype, hope and fear are some of the elements the media uses to sell a story. But how does the media spin influence public perception regarding $\mathrm{SB}$ ? Is there any perception at all? These questions could determine the way, how the public(s) will react towards synthetic biology. The Austrian COSY-Communicating Synthetic Biology project and a study by the Woodrow Wilson Centre for International Scholars, share with us their first results on public perception and the media in this special issue (Torgersen 2009; Kronberger et al. 2009; Cserer and Seiringer 2009; Pauwels 2009).

When it comes to the design and creation of synthetic life, there is one question that pops up again and again, namely: do synthetic biologists play god? Each time this question is dismissed by scientists, it seems to reappear sooner or later. As scientists cannot handle this question it was necessary to involve a theologian. The careful reflections of Dabrock (2009) are very likely to become highly cited in the future.

Apart from the theological side we also need to deal with the ethical issues involved, for example when the categories of "life" and "machine" seem to merge in things like living machines and synthetic organisms (Deplazes and Huppenbauer 2009). Also the creation of protocells, in other words creating living matter out of nonliving matter, will raise new ethical and safety questions as demonstrated by Bedau et al. (2009).

A number of new biosafety issues, such as biosafety engineering and redundant semantic and trophic containment using so-called xeno-nucleic acids as a "biological 
firewall", are also presented here (Schmidt et al. 2009; Marliere 2009).

Biosecurity considerations in SB have so far mainly be discussed in the light of scientific self-regulation or deployment of technical solutions such as the screening of DNA synthesis orders. These are a first start but a more comprehensive approach is needed. How this approach looks like and how the so-called 5P governance strategy may contribute to it, is explained by Kelle (2009).

Among the possible measures to make SB safer and more secure is a code of conduct. Such a code could work better if a professional society of some kind were available to synthetic biologist. The Pros (and Cons) of professionalisation as a governance strategy for SB are envisioned by Weir and Selgelid (2009).

A societal organisation might also help the scientific community to improve the relation between science, social sciences, industry and policy. The current interactions and potential ways to improvement are discussed by Rabinow and Bennett (2009) and Gaisser and Reiss (2009). Part of this relation is the role of ELSI studies (Ethical, Legal and Social Implications) that are located mainly downstream the R\&D process. Rabinow and Bennett (2009) argue that social sciences should be located upstream the R\&D process in order to better analyse and contribute to the societal ramifications of synthetic biology.

Whether it will take 10,20 or 50 years, SB will definitely have a strong impact on the bio-economy. But who will finally benefit from it, only the rich and powerful? A frequently expressed concern by NGOs is that SB is a threat for poor and marginalised people in developing countries that depend on products made from natural resources that could altogether be replaced by cheaper synthetic products. Wellhausen and Mukunda (2009) looked into this potential threat for third world economies in greater detail, by analysing past case studies such as rubber in Malaysia and indigo dyes in India. Their findings show that replacing natural products with synthetic ones, does not automatically lead to the extinction of the natural product.

On a more philosophical level the last paper questions our understanding of SB and the frequently made analogy to computers. This analogy is based on premises where a machine (the cell, cytoplasm), which reproduces over time, runs a program (the genetic code, DNA), which replicates. While the present theory of information has much to say about the program, we almost entirely lack a theory of the information supporting the machine. Danchin (2009) argues that we need to focus our attention also on the machine if we ever want to design and construct biological systems with a certain degree of precision.
In order to make the work presented here widely available, I managed to agree with Springer Publishing to grant Open Choice access to all the papers of this special issue.

Sincerely yours

Markus Schmidt

Guest Editor

\section{In this special issue:}

Bedau MA, Parke EC, Tangen U, Hantsche-Tangen B (2009) Social and ethical checkpoints for bottom-up synthetic biology, or protocells

Cserer A, Seiringer A (2009) Pictures of synthetic biology a reflective discussion of the representation of synthetic biology (SB) in the German-language media and by $\mathrm{SB}$ - experts

Dabrock P (2009) Playing god? Synthetic biology as a theological and ethical challenge

Danchin A (2009) Information of the chassis and information of the program in synthetic cells

Deplazes A, Huppenbauer M (2009) Synthetic organisms-living machines, positioning the products of synthetic biology at the borderline between living and nonliving matter

Gaisser S, Reiss T (2009) Shaping the science-industrypolicy interface in synthetic biology

Kelle A (2009) Ensuring the security of synthetic biology-towards a 5P governance strategy

Kronberger N, Holtz P, Kerbe W, Strasser W, Wagner W (2009) Communicating synthetic biology: from the lab via the media to the broader public

Lorna Weir, Selgelid MJ (2009) Professionalization as a governance strategy for synthetic biology

Marlière P (2009) The farther, the safer: a manifesto for securely navigating synthetic species away from the old living world

Pauwels E (2009) Review of quantitative and qualitative studies on U.S. public perceptions of synthetic biology

Rabinow P, Bennett G (2009) Synthetic biology: ethical ramifications 2009

Schmidt M, Ganguli-Mitra A, Torgersen H, Kelle A, Deplazes A, Biller-Andorno N (2009) A priority paper for the societal and ethical aspects of synthetic biology

Torgersen H (2009) Synthetic biology in societylearning from past experience?

Wellhausen R, Mukunda G (2009) Aspects of the political economy of development and synthetic biology

Open Access This article is distributed under the terms of the Creative Commons Attribution Noncommercial License which permits any noncommercial use, distribution, and reproduction in any medium, provided the original author(s) and source are credited. 